\title{
4 Rechtsgrundlagen
}

Die Feststellung der Rechtsgrundlagen für eine forschende Datenverarbeitung ist für deren Zulässigkeit grundlegend: Zwar muss bei der Auslegung gesetzlicher Regelungen immer der verfassungsrechtliche Rahmen beachtet werden. Für die konkrete Anwendung ist aber vorrangig der Wortlaut der Rechtsgrundlagen bestimmend. Dieser ist auch der Bezugspunkt für dieTransparenz für die Betroffenen, für die Wahrnehmung der Betroffenenrechte, für die Verarbeitungsprozesse beim Verantwortlichen und das zu implementierende Datenschutzmanagement sowie für die stelleninterne, aufsichtliche und gerichtliche Kontrolle.

\subsection{Europäisches und nationales Recht}

Die EU hat ihre Regelungskompetenzen (s.o. Kap. 2.3) mit der Datenschutz-Grundverordnung (DSGVO) wahrgenommen (Verordnung [EU] 2016/679). Die DSGVO ist anders als deren Vorgängerregelung, die europäische Datenschutzrichtlinie 95/46/EG (EG-DSRl) - als Verordnung i.S.v. Art. 288 Abs. 2 AEUV europaweit direkt anwendbar und in allen Teilen verbindlich. ${ }^{105}$

Neben der DSGVO gibt es weitere spezielle europäische Regelungen, die eine Relevanz für die medizinische Forschung und die hierbei stattfindende Datenverarbeitung 
haben. Diese sollen vorliegend nur aufgeführt werden: ${ }^{106}$ die Verordnung über klinische Prüfungen ${ }^{107}$, die Richtlinie menschliches Gewebe und Zellen ${ }^{108}$, die DatenbankRichtlinie $^{109}$ und die Patientenrechte-Richtlinie ${ }^{110}$.

Die DSGVO enthält eine Vielzahl von für die Forschung relevanten Öffnungsklauseln. ${ }^{111}$ Teilweise wird der Begriff „Öffnungsklausel“ abgelehnt, da damit suggeriert werde, dass von den inhaltlichen Vorgaben der DSGVO abgewichen werden könne. Dies ist nicht der Fall. Alternativ wird der Begriff „Spezifizierungsklausel“ verwendet. ${ }^{112}$ Missverständnisse bei Verwendung des inzwischen etablierten Begriffs sind nicht zwingend und in der Praxis auch nicht erkennbar. Deshalb wird dieser Begriff verwendet. Auf der Basis von Öffnungsklauseln erlassene nationale Gesetze dürfen der DSGVO nicht widersprechen, sondern sollen die Vorgaben der DSGVO präzisieren oder konkretisieren. ${ }^{113}$

Bei den Öffnungsklauseln wird unterschieden zwischen solchen, bei denen den Nationalstaaten die Gesetzgebung freigestellt wird (fakultative Öffnungsklauseln), und solchen, bei denen eine Pflicht zur Gesetzgebung besteht (obligatorische Öffnungsklauseln). ${ }^{114}$

Art. 6 Abs. 2, 3 i.V.m. Art. 6 Abs. 1 lit. e DSGVO erlaubt den Mitgliedstaaten die Schaffung von Rechtsgrundlagen für die Ausübung der öffentlichen Gewalt und die Wahrnehmung von Aufgaben im öffentlichen Interesse. Hierzu gehört in jedem Fall die Wahrnehmung der Forschung durch staatliche Hochschulen sowie Maßnahmen von Ministerien und anderen öffentlichen Stellen. ${ }^{115}$ ErwCr. 45 S. 6 stellt zudem klar, dass auch natürliche oder juristische Personen des Privatrechts im öffentlichen Interesse tätig sein können. Als ein Beispiel nennt der Gesetzgeber „gesundheitliche Zwecke, wie die öffentliche Gesundheit oder die soziale Sicherheit oder die Verwaltung von Leistungen der Gesundheitsfürsorge“ ${ }^{116}$ Cenannt werden als weitere öffentliche Interessen „die Verarbeitung für humanitäre Zwecke einschließlich der Überwachung von Epidemien und deren Ausbreitung oder in humanitären Notfällen insbesondere bei Naturkatastrophen oder vom Menschen verursachten Katastrophen“ (ErwCr 46 S. 3) sowie sogar ,wichtige wirtschaftliche oder finanzielle Interessen“" (ErwGr 73 S. 1 am Ende). Das öffentliche Interesse muss ein solches Gewicht haben, dass die Beschränkung des Grundrechts auf Datenschutz verhältnismäßig ist. ${ }^{117}$ Inwieweit Forschung, die im öffentlichen Interesse durchgeführt wird, von dieser Öffnungsklausel erfasst sein soll, ist normativ nicht festgelegt.

106 Ausführlicher Health Ethics Policy Lab, 36ff.

107 Verordnung (EU) Nr. 536/2014 v. 16.04.2014, ABL. EU L 158/1 v. 27.05.2014.

108 Richtlinie 2004/23/EF v. 31.03.2004, ABI. L 102/48 v. 07.04.2004.

109 Richtlinie 96/9/EG v. 11.03.1996, ABl. L 77 20, zuletzt geändert durch Art. 24 ÄndRL (EU) 2019/790 v. 17.04.2019, ABI. L 13092.

110 Richtlinie 2011/24/EU v. 09.03.2011, ABI. L 88/45, betrifft nur die grenzüberschreitende Gesundheitsversorgung; Schneider 2016, $425 \mathrm{ff}$.

111 Roßnagel, ZD 2019, 159; Johannes in Roßnagel 2017, \$ 4 Rn. 83-89.

112 Selmayr/Ehmann in Ehmann/Selmayr, Einführung Rn. 89.

113 Roßnagel in SHS, Art. 6 Rn. 22.

114 Hornung/Spiecker in SHS Einl Rn. 228; Dierks in Dierks/Roßnagel, $9 f$.

115 BKL-R, 220ff.

116 Dierks 2019, 24.

117 Roßnagel in SHS, Art. 6 Abs. 1 Rn. 71. 
Privat finanzierte und von der Wirtschaft ausgeübte Forschung wird nur erfasst, soweit damit konkret eine Aufgabenwahrnehmung im „öffentlichen Interesse“ erfolgt. ${ }^{118}$ Art. 6 Abs. 2 DSGVO sieht im letzten Halbsatz vor, dass die Öffnungsklausel „einschließlich für andere besondere Verarbeitungssituationen gemäßKapitel IX gilt“. Kapitel IX umfasst die Art. 85 bis 91, also auch Art. 89 DSGVO. Daraus wird der Schluss gezogen, dass Art. 6 Abs. 2 DSGVO der Öffnungsklausel des Art. 89 Abs. 2 DSGVO vorgehe. ${ }^{19}$ Darauf kommt es aber nicht an, da sich bzgl. des Umfangs die Regelungsrahmen von Art. 6 Abs. 2 und Art. 89 DSGVO nicht unterscheiden. Auch die Regulierung privilegierter Forschung setzt ein „öffentliches Interesse“ hieran voraus (s.u. Kap. 8.1).

Gemäß Art. 6 Abs. 3 S. 3 DSGVO wird bei einer Verarbeitung im öffentlichen Interesse oder in Ausübung öffentlicher Gewalt die Befugnis eingeräumt, „spezifische Bestimmungen zur Anpassung der Anwendung“ der DSGVO zu erlassen. Diese sollen sich darauf beziehen, „welche Arten von Daten verarbeitet werden, welche Personen betroffen sind, an welche Einrichtungen und für welche Zwecke die personenbezogenen Daten offengelegt werden dürfen und welcher Zweckbindung sie unterliegen, wie lange sie gespeichert werden und welche Verarbeitungsvorgänge und-verfahren angewandt werden dürfen, einschließlich Maßnahmen zur Gewährleistung einer rechtmäßig und nach Treu und Glauben erfolgenden Verarbeitung“.

Diese Öffnungsklausel erweitert die Regelungsmöglichkeiten der Mitgliedstaaten. ${ }^{120}$ Sie geht weiter als Abs. 2, bezieht sich aber, anders als Art. 6 Abs. 2 DSGVO, nicht auf sämtliche DSGVO-Regelungen, sondern nur auf die Rechtsgrundlagen. ${ }^{121}$ Über Art. 6 Abs. 3 DSGVO wird zwar ein Regelungsspielraum geschaffen, doch muss sich dieser im Rahmen der Erlaubnistatbestände der DSGVO halten. Die Regelung präzisiert die inhaltlichen Anforderungen an das spezifizierende nationale Recht. ${ }^{122}$ Die in Art. 89 Abs. 2 BDSG vorgesehenen Privilegierungen in Bezug auf Betroffenenbefugnisse werden von Art. 6 Abs. 3 DSGVO nicht mit umfasst.

Hinsichtlich der Verarbeitung besonderer Datenkategorien (sog. sensitive Daten) ${ }^{123}$ sowie von Berufsgeheimnissen enthält Art. 9 Abs. 2, 3 DSGVO Öffnungsklauseln. ${ }^{124}$ Die Öffnungsklausel in Art. 9 Abs. 2 lit. j DSGVO zur forschenden Verarbeitung von sensitiven Daten verlangt zusätzlich, dass das spezifizierende Recht „den Wesensgehalt desRechts aufDatenschutzwahrt“. Damit wird auf Art. 52 Abs. 1 S. 1 GRCh Bezug genommen, der die Wesensgehaltsgarantie grundrechtlich absichert. Dabei handelt es sich letztlich um eine besondere Betonung und äußere Grenzziehung des Verhältnismäßigkeitsgrundsatzes. ${ }^{125}$

118 Johannes in Roßnagel 2017, § 4 Rn. 83, 85.

119 Roßnagel, Review zum vorliegenden Gutachten v. 02.02.2020, 7, mit Verweis auf BT-Drs. 18/12611, 117 m.w.N.

120 Roßnagel in SHS, Art. 6 Abs. 3 Rn. 36.

121 Roßnagel in SHS, Art. 6 Abs. 3 Rn. 37; differenzierend Frenzel in Paal/Pauly, Art. 6 Rn. 34ff.; a.A. Buchner/Petri in Kühling/Buchner, Art. 6 Rn. 195: gleicher Regelungsumfang; unentschieden Schulz in Gola, Art. 6 Rn. 172.

122 Dierks 2019, 24; Reimer in Sydow, Art. 6 Rn. 24; Schwartmann/Jaquemain in SJTK, Art. 6 Rn. 168; a.A. Kramer in Auernhammer, Art. 6 Rn. 62: implizite Festlegung des Zwecks genügt.

123 ErwGr 10, 5 spricht von „sensiblen Daten“; demgegenüber wird hier der Begriff „sensitive Daten“ verwendet, mit dem die besondere Schutzbedürftigkeit besser zum Ausdruck kommt.

124 Kühling, 23ff.; Weichert in Kühling/Buchner, Art. 9 Rn. 138ff.; zur nationalen Relevanz bei der Forschung Geminn RDV 2019, 116ff.

125 Petri in SHS, Art. 9 Rn. 76; EuGH 06.10.2015 - C-362/14 (Safe Harbor), Rn. 94, NJW 2015, 3157. 
Jeweils eine umfassende fakultative Öffnungsklausel enthalten schließlich Art. 85 DSGVO für die wissenschaftliche Kommunikation und Art. 89 Abs. 2 DSGVO für „Bedingungen und Garantien“ bei Forschungs- und Statistikzwecken sowie hinsichtlich der erforderlichen Ausnahmen von Betroffenenrechten. ${ }^{126}$ Voraussetzung für das Zurücktreten von Betroffeneninteressen ist, dass ein öffentliches Interesse an der Durchführung eines Forschungsvorhabens besteht und davon ausgegangen werden kann, dass verlässliche wissenschaftliche Ergebnisse zu erwarten sind. ${ }^{127}$ Von Art. 85 DSGVO nicht mit umfasst sind Informationsbegehren von Betroffenen oder Kontrollbegehren im Rahmen der Datenschutzaufsicht. ${ }^{128}$

Einige der Öffnungsklauseln richten sich nicht nur an die Mitgliedstaaten, sondern fakultativ auch an die Union. Dies gilt insbesondere für die Regelung der Verarbeitung von Gesundheitsdaten als eine Form der sensitiven Daten (Art. 9 Abs. 2 lit. a, b, g, h, i, j DSGVO) sowie von Berufsgeheimnissen (Art. 9 Abs. 3 DSGVO). In diesen Bereichen ist die EU befugt, den Mitgliedstaaten verbindliche Vorgaben zu machen. Von diesen Möglichkeiten hat die EU im Bereich der Verarbeitung von Gesundheitsdaten bzw. im Medizinbereich bisher keinen Gebrauch gemacht.

Die Intentionen der Öffnungsklauseln unterscheiden sich. Teilweise sind sie einer fehlenden Regelungskompetenz der EU geschuldet (so Art. 85 DSGVO für den Medienbereich). ${ }^{129}$ Teilweise liegt der Grund für sie in nationalen Regelungstraditionen und/ oder in der fehlenden Bereitschaft der Mitgliedstaaten, sich auf EU-Ebene zu einigen. ${ }^{130}$ Deutschland legte besonderen Wert darauf, dass das teilweise stark spezifizierte Datenschutzrecht und dort insbesondere das Sozialrecht (Art. 9 Abs. 2 lit. b DSVO), das Recht der öffentlichen Verwaltung und die Regelungen des Gesundheitswesens und der Berufsgeheimnisträger (Art. 9 Abs. 2 lit. b, h, i u. Abs. 3, Art. 9o) beibehalten werden können. ${ }^{131}$ Während die EU-Kommission für die Bereiche Forschung, Archive und Statistik zunächst einen weiten Ausgestaltungsvorschlag machte, wurde der Spielraum in den Art. 89 Abs. 2-4 DSGVO letztlich darauf beschränkt, dass Betroffenenrechte die Verarbeitungszwecke zumindest ernsthaft beeinträchtigen. ${ }^{132}$

Die für den Bereich der medizinischen Forschung relevanten Öffnungsklauseln in

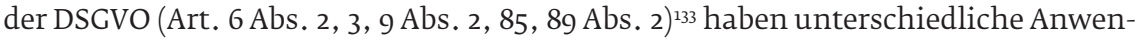
dungsbereiche und Voraussetzungen und gewähren damit den Mitgliedstaaten unterschiedliche Regelungskompetenzen. Rechtfertigen unterschiedliche Öffnungsklauseln eine nationale Regelung, so ergänzen sie sich gegenseitig. ${ }^{134}$ Doch muss auch bei der Anwendung der spezifizierenden Normen in jedem Fall der von der DSGVO vorgesehene Regelungsrahmen beachtet werden. Bei der Nutzung der Öffnungsklauseln muss zudem die Verhältnismäßigkeit der Eingriffe für den verfolgten Zweck ge-

126 Geminn, DuD 2018, 642.

127 Dierks 2019, 30; BKL-R, 205.

128 Johannes in Roßnagel 2017, $\$ 4$ Rn. 89.

129 Albrecht//otzo, Teil 9 Rn. 5.

130 Albrecht/Jotzo, Teil 1 Rn. 16, 18

131 Albrecht//otzo, Teil 1 Rn. 24, Teil 3 Rn. 46, 58.

132 Albrecht/Jotzo, Teil 9 Rn. 8.

133 Dierks 2019, $23 f f$.

134 A.A. wohl Richter in Roßnagel 2018, § 7 Rn. 163, Roßnagel, Review zum vorliegenden Gutachten v. 02.02.2020, $6 f$. 
wahrt bleiben. Dies hat zur Folge, dass ,angemessene und spezifische Maßnahmen zur Wahrung der Crundrechte und Interessen der betroffenen Person " vorgesehen sein müssen. ${ }^{135}$

National nicht abdingbar sind die in Art. 5 DSGVO festgelegten Datenschutzgrundsätze. Zur nationalen Disposition stehen kann daher auch nicht die generelle in Art. 5 Abs. 1 lit. b DSGVO gewährleistete Zweckprivilegierung. Nach anderer Ansicht soll die Zweckvereinbarkeitsfiktion nur gelten, wenn es keine Regelung eines Mitgliedsstaates zur Zweckänderung aufgrund einer einschlägigen Öffnungsklausel gibt. Eine solche könne in Art. 6 Abs. 3 S. 3 i.V.m. Art. 6 Abs. 1 lit. e DSGVO gesehen werden. ${ }^{136}$ Dem kann nicht gefolgt werden, da danach die Regelung privilegierter Forschungsprojekte völlig der Disposition der nationalen Gesetzgeber überlassen bliebe. Dies kann nicht die Intention des EU-Gesetzgebers gewesen sein. Beschränkt man die Öffnungsklausel des Art. 6 Abs. 3 S. 3 DSGVO ausschließlich auf öffentliche Stellen, so hätte dies zur Folge, dass private privilegierte Forschung immer auf Art. 5 Abs. 1 lit. b DSGVO zurückgreifen könnte, nicht aber die von öffentlicher Hand betriebene Forschung. Es ist Ziel der Privilegierung, EU-weit die Nutzung von personenbezogenen Daten für die Forschung einheitlich zu erleichtern, ohne dass dies von Mitgliedstaaten wieder zurückgenommen werden kann.

Die Anwendbarkeit von Bundes- oder Landesrecht bei der Umsetzung der Öffnungsklauseln orientiert sich weitgehend an der Art der jeweils die personenbezogenen Daten verarbeitenden Stellen. So gilt für Stellen des Bundes (z.B. Forschungseinrichtungen, Bundeswehrklinik), für Sozialleistungsträger und für nicht-öffentliche (private) Stellen Bundesrecht.

Für Stellen der Länder sowie der Kommunen (Hochschulen, öffentliche Krankenhäuser, Gesundheitsbehörden) ist Landesrecht anwendbar, soweit nicht für spezifische Fragestellungen ein Bundesgesetz erlassen worden ist. In Bezug auf das Gesundheitswesen und die Forschung enthält das Grundgesetz (GG) keine spezifischen Gesetzgebungszuständigkeiten, sodass diese gemäß Art. 70 GG weitgehend bei den Ländern liegt. Bundeszuständigkeiten bestehen in Bezug auf die Privatwirtschaft (Art. 74 Abs. 1 Nr. 11 i.V.m. Art. 72 Abs. 1 GG), die Bundesverwaltung (Art. 87 GG) sowie das gesamte Sozialrecht (Art. 74 Abs. 1 Nr. 12 i.V.m. Art. 72 Abs. 1 GG, s.o. Kap. 2.4).

Landesrecht ist bei öffentlichen Stellen der Länder und der Kommunen im Ergebnis nicht oder nur sehr eingeschränkt anwendbar, soweit die Forschung als Wettbewerbstätigkeit einzustufen ist. ${ }^{1{ }^{37}}$ In diesem Fall tritt an die Stelle des Landesrechts das BDSG. Ob eine Wettbewerbstätigkeit anzunehmen ist, muss im Einzelfall beurteilt werden. Gründe hierfür können darin liegen, dass ein enger Zusammenhang mit einem primären Behandlungszweck besteht und die Behandlung im Wettbewerb erbracht wird. Aber auch die Forschungstätigkeit selbst steht im Wettbewerb, soweit diese in Konkurrenz zu anderen Einrichtungen um Mittelzuweisungen und valide Ergebnisse steht. ${ }^{138}$

135 So explizit Art. 9 Abs. 2 lit. j, inhaltlich ebenso Art. 89 DSGVO.

136 Roßnagel, Review des vorliegenden Gutachtens, 02.02.2020, $10 \mathrm{f}$.

$137 \S 2$ Abs. 6 LDSG BW, Art. 1 Abs. 3 BayDSG, $\$ 2$ Abs. 6 BlnDSG, $§ 2$ Abs. 3 BbgDSG, $\ 2$ Abs. 3 BremDSGVOAG, $\S 2$ Abs. 3 HmbDSG, $\$ 2$ Abs. 2 HDSIG, $\$ 2$ Abs. 5 DSG MV, $\$ 1$ Abs. 4 NDSG, $\$ 5$ Abs. 5 Nr. 4 DSG NRW, $\$ 2$ Abs. 4 LDSG RP, \ 2 Abs. 3 SDSG, \2 Abs. 3 SächsDSG, \2 Ab. 7 Nr. 1 DSAG LSA, \2 Abs. 4 LDSG SH.

138 Schneider 2015, $91 \mathrm{f}$. 


\subsection{Datenschutz-Grundverordnung}

Die seit dem 25.05.2018 direkt anwendbare und wirkende europäische DatenschutzGrundverordnung (DSGVO) verfolgt das Ziel, das Datenschutzrecht an die technische Entwicklung anzupassen, insofern zu modernisieren und dadurch den Grundrechtsschutz zu verbessern (ErwGr 1, 2, 4, 6), das Datenschutzrecht zu harmonisieren und dadurch einheitliche Bedingungen für die Datenverarbeitung im Binnenmarkt zu schaffen (ErwGr 5, 7, 8). Die Zulässigkeit der Datenverarbeitung - auch für Forschungszwecke - ergibt sich aus den allgemeinen Regelungen der DSGVO, also aus Art. 5 und 6. ${ }^{139}$

Hinsichtlich der Verarbeitung von besonderen Datenkategorien (sensitive Daten), also z.B. von Gesundheitsdaten, werden Art. 9 Abs. 2 DSGVO sowie darauf basierende weitere Normen als eigenständige Rechtsgrundlagen angesehen. ${ }^{140}$ Dies wird damit begründet, dass die Vorgaben von Art. 6 DSGVO zu unspezifisch für die Verarbeitung von sensitiven Daten seien. Außerdem sei unklar, in welcher Kombination aus Tatbestandsvoraussetzungen des Art. 6 Abs. 1 DSGVO mit solchen des Art. 9 Abs. 2 DSGVO eine wirksame Legitimation zu sehen sei. ${ }^{141}$ Die Vorgaben Art. 6 Abs. 1 DSGVO sind tatsächlich sehr unspezifisch. Sie sind aber in mancher Hinsicht spezifischer als die in Art. 9 Abs. 2 DSGVO, z.B. wenn es um das Abwägungserfordernis in Art. 6 Abs. 1 lit. f DSGVO geht. Richtig ist, dass sich die Art. 6 und 9 DSGVO gegenseitig ergänzen. Die Unsicherheit über die Rechtsgrundlagen besteht schon über den Umstand, dass diese oft auf den Öffnungsklauseln in Art. 6 Abs. 1 oder Art. 9 Abs. 2 DSGVO beruhen. Würde zwischen Art. 6 Abs. 1 und Art. 9 Abs. 2 DSGVO ein Ausschlussverhältnis gesehen, so bestünde die Möglichkeit, dass sensitive Daten einen geringeren Schutz genössen als nicht-sensitive Daten. Zudem ließe sich dogmatisch schwer erklären, weshalb Art. 6 Abs. 4 DSGVO bei einer sensitiven Datenverarbeitung anzuwenden wäre. ${ }^{142}$

Mit den Regelungen in Art. 9 Abs. 2 DSGVO werden die sehr allgemeinen Vorgaben des Art. 6 konkretisiert und eingeengt. ${ }^{143}$ Da die Art. 6 und 9 DSGVO jeweils Öffnungsregelungen enthalten, die eine weitere Konkretisierung der Vorgaben ermöglichen, kann durch diese Rechtsgrundlagen (z.B. im BDSG oder im spezifischen Datenschutzrecht) eine weitere Präzisierung und Einengung erfolgen. Deren Anwendung muss sich aber im Rahmen der Art. 6 und 9 DSGVO halten. ${ }^{144}$ Der Europäische Datenschutzausschuss benennt daher sowohl Art. 6 in seinen besonderen Ausprägungen wie auch Art. 9 DSGVO gemeinsam als Rechtsgrundlage. ${ }^{145}$ Durch die Privilegierung für Forschungszwecke in der DSGVO erfolgen auch Befugnisausweitungen in Bezug auf die Zweckänderung oder die Betroffenenrechte. Maßstab ist der Grundrechtsein-

139 Albrecht/Jotzo, Teil 3 Rn. 72; Geminn, DuD 2018, 641.

140 Dierks 2019, 25.

141 Dierks 2019, 25.

142 Dafür Dierks 2019, 25f.; weitergehend z.B. Schiff in Ehmann/Selmayr, der unter Art. 9 DSGVO Art. 6 Abs. 4 DSGVO nicht zur Anwendung bringen will (s.u. Kap. 4.4).

143 Kühling, 45; Weichert in Kühling/Buchner, Art. 9 Rn. 4; Petri in SHS, Art. 9 Rn. 3; Golla in Specht/Mantz, § 23 Rn. 24, 26; Werkmeister/Schwaab CR 2019, 87.

144 Golla in Specht/Mantz, § 23 Rn. 34f.; Piper DANA 2019, 72; zu § 27 BDSG Johannes/Richter, DuD 2017, 302; Werkmeister/Schwaab CR 2019, 89; Krohm in Gola/Heckmann, § 27 Rn. $7 f$.

145 EDSA, 5f., 8f. (Rn. 10-13, 25-28). 
griff in das informationelle Selbstbestimmungsrecht der Betroffenen in Abwägung mit der Ermöglichung freier Forschung.

Ein zentraler Abwägungsaspekt ist, wie hoch das Risiko einer Zweckentfremdung, also einer übermäßig eingreifenden Zweckänderung, ist. Hierbei spielen technische, organisatorische wie auch rechtliche Erwägungen eine Rolle. Solange kein gesetzlich garantiertes Forschungsgeheimnis vor einem Datenzugriff und einer Nutzung durch Sicherheitsbehörden oder sonstige administrativ tätige Stellen bewahrt, ist dies im Rahmen der Abwägung mit zu berücksichtigen. ${ }^{146}$

Bei der Abwägung zwischen Datenschutz, weiteren Betroffenengrundrechten und der Forschungsfreiheit ist von Bedeutung, aus welcher Quelle die personenbezogenen Informationen stammen. Generell gilt zwar, dass es in Zeiten der automatisierten Datenverarbeitung kein belangloses Datum gibt. ${ }^{147}$ Dessen ungeachtet hängt die Intensität der informationellen Eingriffe davon ab, ob Daten zum Kernbereich privater Lebensgestaltung gehören, aus dem Intimbereich stammen, aus sozialen Beziehungen der Betroffenen oder aus der Öffentlichkeitssphäre. ${ }^{148}$

Daten, die der Öffentlichkeitssphäre zuzuordnen sind und öffentlich zugänglich sind, so wie dies bei Daten im Internet der Fall ist, unterliegen also auch dem Persönlichkeitsschutz, sodass bei deren Verarbeitung eine Abwägung zwischen den Betroffenenbelangen und den Verarbeitungsinteressen nötig ist. ${ }^{149}$ Art. 9 Abs. 2 lit. e DSGVO setzt für die Zulässigkeit der Verarbeitung besonderer Datenkategorien voraus, dass die öffentliche Zugänglichkeit der Daten auf einer offensichtlich bewussten Handlung des Betroffenen basiert. ${ }^{150}$ An dem Abwägungserfordernis ändert auch der Umstand der generellen Privilegierung von Forschungszwecken nichts. ${ }^{151}$ Zwar geht die DSGVO davon aus, dass Forschung mit personenbezogenen Daten grundsätzlich für die Gemeinschaft nützlich ist und deshalb geringeren Beschränkungen unterworfen wird. Das Ergebnis einer Interessenabwägung kann aber dennoch sein, dass wegen der damit verbundenen Eingriffe in die Grundrechte der Betroffenen oder sich ergebender Risiken ein konkretes Projekt nicht zugelassen werden kann (s.u. Kap. 12.8).

\subsection{Nationales Datenschutzrecht}

Mit Wirkung zum 25.05.2018 wurde das allgemeine Datenschutzrecht in Deutschland novelliert und das bisherige Bundesdatenschutzgesetz (BDSG) durch eine völlig neue Regelung ersetzt. ${ }^{152}$ Die wesentlichen Regelungen in den Sozialgesetzbüchern (SCB), also insbesondere $\mathbb{3} 35$ SGB I und die $\$ \$ 67 f f$. SCB X, wurden ebenso angepasst. ${ }^{153}$ Weitere umfangreiche Anpassungen des nationalen Datenschutzrechts erfolgten im

146 Tinnefeld/Buchner/Petri/Hof, Einführung in das Datenschutzrecht, 7. Aufl. 2020, Rn. $506 f$.

147 BVerfG 15.12.1983 - 1 BvR 209/83 u.a., NJW 1984, 422.

148 Weichert in DKWW, Einl. Rn. 11f.

149 EuGH 14.05.2014 - C-131/12, Rn. 81f.; EDPS, 18.

150 Petri in SHS, Art. 9 Rn. $57 f$.

151 Golla in Specht/Mantz, \23 Rn. 39; relativierend Golla/von Schönfeld, K\&R 2019, 19.

152 Art. 1 des Datenschutz-Anpassungs- und Umsetzungsgesetz EU (DSAnpUG-EU) v. 30.06.2017, BGBI. I S. 2097.

153 Art. 19, 24 des Gesetzes zur Änderung des Bundesversorgungsgesetzes und anderer Vorschriften v. 17.07.2017, BGBI. I S. 2541; dazu Roßnagel/Geminn in Dierke/Roßnagel, 141. 
Herbst 2019. ${ }^{154}$ Mit den nationalen Überarbeitungen wurden insbesondere gemäß den Öffnungsklauseln in der DSGVO konkretisierende Regelungen erlassen. Dabei hat der Gesetzgeber das innovative Potenzial der DSGVO nicht ausgeschöpft, sondern seine Kompetenzen weitgehend dafür genutzt, bisherige Regelungen beizubehalten, ja sogar die Möglichkeiten zur Verarbeitung zu erweitern sowie Betroffenenrechte zu beschränken. ${ }^{155}$

Während der Bundesgesetzgeber die Umsetzungsregelungen zur DSGVO relativ frühzeitig verabschiedete, schafften die meisten Bundesländer diese Anpassung in Bezug auf das allgemeine Datenschutzrecht erst kurz vor oder nach dem 25.05.2018. Einige Anpassungen wurden bis heute nicht in Angriff genommen, so etwa das allgemeine Datenschutzgesetz von Sachsen-Anhalt oder Regelungen in den Landeskrankenhausgesetzen ${ }^{156}$. Auch hier beschränkten sich die Parlamente zumeist auf eine formelle Anpassung, ohne die inhaltlichen europäischen Neuorientierungen zu übernehmen. Dies gilt insbesondere auch für die Forschungsklauseln in den Landesdatenschutzgesetzen.

Wegen der eher konservativen deutschen Umsetzung der DSGVO erfolgten nur wenige strukturelle Änderungen gegenüber dem zuvor geltenden Recht, was eine Fortsetzung bisheriger Verarbeitungspraktiken erleichtert. Dies gilt auch für die Verarbeitung für Forschungszwecke, für die in der DSGVO weitestgehend Öffnungsklauseln zur Anwendung kommen. ${ }^{157}$ Zugleich wird mit diesem Vorgehen der Ansatz der DSGVO, eine möglichst umfassende Modernisierung und Anpassung an neue technische, ökonomische und soziale Entwicklungen und eine Harmonisierung vorzunehmen, konterkariert.

Von der Änderung des Datenschutzrechts weitgehend unabhängig erfolgte $2017 \mathrm{mit}$ dem Gesetz zur Neuregelung des Schutzes von Geheimnissen bei der Mitwirkung an der Berufsausübung schweigepflichtiger Personen eine Überarbeitung des $\mathbb{S} 203$ StGB, mit dem die Möglichkeiten für Schweigepflichtige erweitert wurden, „sich im Rahmen ihrer beruflichen und dienstlichen Tätigkeiten ohne (straf-)rechtliches Risiko der Mitwirkung dritter Personen zu bedienen." (s.u. Kap. 6.6) ${ }^{158}$

\subsection{Forschungsregelungen in der DSGVO}

Die DSGVO verfolgt gegenüber dem bisher geltenden europäischen wie nationalen Recht einen völlig neuen Ansatz, indem sie für die Forschung weitgehende Ausnahmeregelungen enthält mit einer Privilegierung des Verfolgens von Forschungszwecken gegenüber sonstigen Verarbeitungszwecken. Dies gilt generell gemäß Art. 89 Abs. 2 und speziell für die zweckändernde Datennutzung (Art. 5 Abs. 1 lit. b), die Begrenzung der Speicherdauer (Art. 5 Abs. 1 lit. e), die Zulässigkeit der Verarbeitung besonderer Kategorien personenbezogener Daten (Art. 9 Abs. 2 lit. j), die Benachrich-

154 Zweites Gesetz zur Anpassung des Datenschutzrechts an die Verordnung (EU) 2016/679 und zur Umsetzung der Richtlinie (EU) 2016/680 (Zweites Datenschutz-Anpassungs- und Umsetzungsgesetz EU - 2. DSAnpUG-EU) v. 20.11.2019, BGBl. I S. 1626, zuvor BT-Drs. 19/4674.

155 Roßnagel/Geminn in Dierks/Roßnagel, 141; Roßnagel, DuD 2017, 277.

156 Dierks 2019, $37 f f$.

157 Art. 6 Abs. 2, 9 Abs. 2 lit. j, 85 Abs. 1, 89 DSGV0.

158 G. v. 30.10.2017, BGBl. I S. 3618; BT-Drs. 18/11936, 17. 
tigungspflicht der Betroffenen, wenn personenbezogene Daten nicht bei ihnen erhoben werden (Art. 14 Abs. 5 lit. b), die Löschungspflicht bzw. das Recht auf Vergessenwerden (Art. 17 Abs. 3 lit. d) sowie das Widerspruchsrecht (Art. 21 Abs. 6). ${ }^{159}$

Es ist umstritten, ob sich der Verantwortliche für die Forschungsnutzung auf die Rechtsgrundlage des Primärzwecks berufen kann. ${ }^{160}$ Teilweise wird die Ansicht vertreten, dass die in Art. 5 Abs. 1 lit. b DSGVO enthaltene Formulierung, dass eine Weiterverarbeitung für wissenschaftliche Forschungszwecke nicht unvereinbar mit dem ursprünglichen Zweck sei, bedeutet, dass die forschende Verarbeitung sich auf die Grundlage der Erstverarbeitung stützen könne. ${ }^{161}$ Diese Ansicht stützt sich auf ErwGr 5o S. 2, wo es in Bezug auf die Regelung zur Zweckänderung in Art. 6 Abs. 4 DSGVO heißt:

„In diesem Fall ist keine andere gesonderte Rechtsgrundlage erforderlich als diejenige für die Erhebung der personenbezogenen Daten.“

Erwägungsgründen kommt keine verbindliche normative Kraft zu. ${ }^{162}$ Die Formulierung von ErwGr 5o S. 2 wird aus der Entstehungsgeschichte erklärt, wonach in der Auseinandersetzung über die Reichweite der Zweckbindung dieser Satz stehengeblieben ist, während normativ die Zweckbindung in Art. 6 Abs. 4 DSGVO eingeschränkt wurde. ${ }^{163}$ Der Europäische Datenschutzausschuss (EDSA) beruft sich zwar auf den ErwGr. 5o S. 2 und akzeptiert, dass es keiner neuen Rechtsgrundlage für eine Sekundärnutzung bedarf. ${ }^{164}$ Er sieht aber das Risiko, dass über eine Sekundärnutzung schutzwürdige Betroffeneninteressen verletzt sein können, und meint, dass dies „künftig besondere Aufmerksamkeit und Anleitung seitens des EDSA“ erfordert. ${ }^{165}$ Der Europäische Datenschutzbeauftragte bestätigt, dass der Erwägungsgrund nicht dazu führen kann, dass der Vereinbarkeitstest von Art. 6 Abs. 4 DSGVO unterlassen wird. ${ }^{166}$

Die DSGVO enthält keine Freistellungen von der Zweckbindung, sondern lediglich eine Flexibilisierung. Dies gilt für Art. 6 Abs. 4 DSGVO. Entsprechendes gilt für Art. 5 Abs. 1 lit. b DSGVO für die Weiterverarbeitung für Forschungszwecke. Das sich an Art. 8 GRCh ausrichtende Konzept der DSGVO basiert darauf, dass zweckbezogene Rechtsgrundlagen gefordert werden und zweckändernde Verarbeitungen einer spezifischen Rechtsgrundlage bedürfen. Dies gilt erst recht für die Forschungsprivi-

159 Dierks 2020, 7ff.; Schäfer in Kipker/Voskamp, 334f.; Geminn DuD 2018, 641f.; zumindest missverständlich ist die Formulierung von Golla in Specht/Mantz, \$23 Rn. 4, wonach die DSGVO „keine umfassende Privilegierung wissenschaftlicher Zwecke" enthält.

160 BT-Drs. 18/11325, 99; Richter, DuD 2015, 735; ders., DuD 2016, 584f.; Graf von Kielmansegg in TMF, 102; Golla in Specht/Mantz, \ 23 Rn. 56; Herbst in Auernhammer, \ 27 Rn. 15; Wolff in Schantz/Wolff, Rn. 411, 413; ErwGr 50, 2 u. 5 sagen nichts anderes aus; zweifelnd dazu Hornung/Hofmann, ZD-Beilage 4/2017, 7f.; neutrale Darstellung bei BKL-R, 234ff.; richtig Kühling/Buchner-Herbst, Art. 5 Rn. 54; Graf von Kielmansegg in TMF, 102.

161 So Schulz in Gola, Art. 6 Rn. 185ff.; Frenzel in Paal/Pauly, Art. 5 Rn. 31; Roßnagel/Geminn in Dierks/Roßnagel.

162 Selmayr/Ehmann in Ehmann/Selmayr, Einführung Rn. 97; Hornung/Spiecker in SHS, Einl Rn. 270; Weichert in DWWS, Einl DSGVO Rn. 39.

163 Herbst in Kühling/Buchner Art. 5 Rn. 49; Schantz, NJW 2016, 1844; a.A. Monreal ZD 2016, 510.

164 Zum Nutzen der Sekundärnutzung von Patientendaten Zenker/Krawczak/Semler in TMF, 9.

165 EDSA, 9 (Rn. 31), vgl. auch EDPS, $7 f$.

166 EDPS, 23. 
legierung, auf die sich der Erwägungsgrund zu Art. 6 Abs. 4 nicht explizit bezieht. ${ }^{167}$ Art. 5 Abs. 1 lit. b DSGVO schließt nur die Unvereinbarkeit der Zwecke aus und begründet nicht eine Vereinbarkeit in jedem Fall. Als Rechtsgrundlage sind also die spezifischen Regelungen heranzuziehen, soweit Öffnungsklauseln bestehen. Anderenfalls ist direkt auf Art. 6 und Art. 9 DSGVO zurückzugreifen. Bei Anwendung der spezifischen Regelungen sind die Wertungen der Art. 6 (und evtl. Art. 9) sowie die Privilegierung in Art. 5 Abs. 1 lit. b DSGVO als höherrangiges Rahmenrecht zu beachten. ${ }^{168}$

Eine andere Sicht würde im Ergebnis dazu führen, dass für Forschungszwecke in jedem Fall auf die ursprüngliche Rechtsgrundlage zurückgegriffen werden könnte und innerhalb von Forschungsnutzungen eine Zweckbindung ausgehebelt würde. ${ }^{169}$ Unklar wäre, in welchem Verhältnis die Rechtsgrundlage der primären Verarbeitung zu spezifizierenden Forschungsklauseln stünde. Würden Daten von einem Verantwortlichen zusammengeführt, die aus unterschiedlichen Quellen mit unterschiedlicher Rechtsgrundlage stammen, wäre unklar, welcher Rechtsgrund anwendbar sein soll. Eine einwilligungsbasierte Forschungsnutzung würde z.B. grundsätzlich die Datennutzung für beliebige sonstige Forschungszwecke eröffnen. Tatsächlich gilt der Zweckbindungsgrundsatz auch innerhalb der Forschung (s.u. Kap. 7). Die Verarbeitungserlaubnis setzt in jedem Fall das Bestehen von angemessenen Garantien voraus (vgl. Art. 89).

Wird dies anerkannt, so reduziert sich die Frage nach der Rechtsgrundlage darauf, welche Norm als Rechtsgrundlage benannt wird. Im Interesse der Betroffenentransparenz wie auch der Rechtsklarheit für alle anderen Beteiligten sollte dies jeweils die anwendbare spezifische die Forschungsverarbeitung regelnde Norm sein. Zu diesem Ergebnis kommt man auch, wenn auf die Rechtsgrundlage des Primärzwecks zurückgegriffen werden kann.

In Art. 85 DSGVO, der die „Verarbeitung und Freiheit der Meinungsäußerung und Informationsfreiheit“ regelt, werden die Mitgliedstaaten zum Erlass von Rechtsvorschriften verpflichtet, wobei die Verarbeitung „,von wissenschaftlichen, künstlerischen oder literarischen Zwecken“ eingeschlossen wird. In Art. 89 DSGVO werden Vorgaben gemacht für die

„Garantien und Ausnahmen in Bezug auf die Verarbeitung zu im öffentlichen Interesse liegenden Archivzwecken, zu wissenschaftlichen oder historischen Forschungszwecken und zu statistischen Zwecken“.

Während die DSGVO fast durchgängig den Begriff „wissenschaftliche (und historische) Forschungszwecke“ verwendet, wenn es um eine spezifische Forschungsprivilegierung geht (Art. 5 Abs. 1 lit. b, e, 9 Abs. 2 lit. j, 14 Abs. 5 lit. b, 17 Abs. 3 lit. c, 21 Abs. 6, 89 Abs. 2), wird in Art. 85 DSGVO nur der Begriff der „wissenschaftlichen Zwecke" verwendet, der auch in Art. 6 Abs. 1 lit. b EG-DSRl Verwendung fand. Art. 85 DSGVO enthält eine Öffnungsklausel, mit welcher der Datenschutz mit der Meinungsfreiheit und der Informationsfreiheit ,in Einklang“ gebracht werden soll (eben-

167 Herbst in Kühling/Buchner Art. 5 Rn. 49; so im Ergebnis auch Roßnagel in SHS, Art. 6 Abs. 4 Rn. 41.

168 Piper DANA 2019, 72; Wiebe, 542f. m.w.N.

169 So tatsächlich Johannes in Roßnagel 2017, \$ 4 Rn. 64. 
so ErwGr 165 S. 7). In Art. 85 Abs. 2 DSGVO ist bei der Verarbeitung von Personendaten eine Privilegierung im Interesse von Meinungsäußerung und Informationsfreiheit vorgesehen. Diese unterscheidet sich von den expliziten Forschungsprivilegierungen der DSGVO - zumindest teilweise - sowohl im Inhalt wie im Zweck: Gemeinsames Ziel ist der aufgeklärte demokratische Diskurs. Bei den Forschungsprivilegierungen in der DSGVO geht es um die Suche nach objektivierbarer Wahrheit. Vom Schutz der Meinungsäußerung werden auch stark subjektive Wertungen erfasst. Bei der Forschung werden teilweise quantitativ und qualitativ massive Eingriffe in die informationelle Selbstbestimmung vieler Betroffener erlaubt, was mit einer strengen Zweckbindung, einer Pflicht zur Anonymisierung und weiteren Garantien eingefangen werden soll. ${ }^{170}$ Im Interesse der objektivierbaren Wahrheit werden bei der Forschung hohe Anforderungen an Methodik und Transparenz (Art. 89 Abs. 2 DSGVO) gestellt. Demgegenüber treffen Eingriffe nach Art. 85 DSGVO zumeist Einzelpersonen in bestimmten Zusammenhängen. Zugleich gibt es keine einschränkenden Vorgaben für die Verarbeitung.

Art. 85 und 89 DSGVO verfolgen selbst im wissenschaftlichen Bereich teilweise unterschiedliche Zielrichtungen. Art. 85 DSGVO ist insofern weiter und erfasst zweifellos auch die nicht privilegierte Forschungstätigkeit, also Aktivitäten, die z.B. wegen ihrer Anwendungsorientierung, wegen fehlender Transparenz oder einer übermäßig spekulativen Methodik nicht nach den spezifischen Regelungen der DSGVO privilegiert sind. Während Art. 89 DSGVO seinen Schwerpunkt in der Forschungsfreiheit generell hat, liegt der Schwerpunkt von Art. 85 DSGVO auf dem kommunikativen Aspekt der Wissenschaft. ${ }^{171}$ Dieser betrifft nicht nur die Kommunikation über die Art der Forschung und deren Ergebnisse, sondern auch den Zugang zu Forschungsgrundlagen (Datenerhebung, Informationsfreiheit) ${ }^{172}$, wovon Art. 89 nur einen Teilbereich abdeckt. Hierfür schien der Begriff „wissenschaftliche Zwecke“ zu weit und wurde ersetzt durch den der „wissenschaftlichen Forschungszwecke“. ${ }^{173}$ Die weiteren Privilegierungen des Art. 85 DSGVO dürfen nicht dazu verwendet werden, die höheren Anforderungen des Art. 89 DSGVO zu umgehen. ${ }^{174}$

\subsection{Deutsche Forschungsregelungen}

Das Bundesdatenschutzgesetz enthält nun in $\$ 27$ eine Regelung zur „Datenverarbeitung zu wissenschaftlichen oder historischen Forschungszwecken und zu statistischen Zwecken“, mit der die Öffnungsklauseln der DSGVO umgesetzt werden sollen. Eine Konkretisierung der generellen Zweckänderungsbefugnis nach Art. 5 Abs. 1 lit. b DSGVO erfolgt dabei nicht, sondern nur eine in Bezug auf sensitive Daten (Abs. 1, 3). Abs. 2 enthält Ausnahmeregelungen zu den Betroffenenrechten:

170 Caspar in SHS, Art. 89 Rn. 2.

171 EDPS, 10f.; Golla in Specht/Mantz, § 23 Rn. 6; Hornung/Hofmann, ZD-Beilage 4/2017, 12; SHS-Dix, Art. 85 Rn. 19.

172 Buchner/Tinnefeld in Kühling/Buchner, Art. 85 Rn. 21.

173 Albrecht/Jotzo, Teil 3 Rn. 71; zu den ausdifferenzierten Begriffen im Detail Roßnagel/Geminn in Dierks/Roßnagel, $210 f f$.

174 EDPS, 11. 
„(1) Abweichend von Artikel g Absatz 1 der Verordnung (EU) 2016/679 ist die Verarbeitung besonderer Kategorien personenbezogener Daten im Sinne des Artikels g Absatz 1 der Verordnung (EU) 2016/679 auch ohne Einwilligung für wissenschaftliche oder historische Forschungszwecke oder für statistische Zwecke zulässig, wenn die Verarbeitung zu diesen Zwecken erforderlich ist und die Interessen des Verantwortlichen an der Verarbeitung die Interessen der betroffenen Person an einem Ausschluss der Verarbeitung erheblich überwiegen. Der Verantwortliche sieht angemessene und spezifische Maßnahmen zur Wahrung der Interessen der betroffenen Person gemäß $\$ 22$ Absatz 2 Satz 2 vor.

(2) Die in den Artikeln 15, 16, 18 und 21 der Verordnung (EU) 2016/679 vorgesehenen Rechte der betroffenen Person sind insoweit beschränkt, als diese Rechte voraussichtlich die Verwirklichung der Forschungs- oder Statistikzwecke unmöglich machen oder ernsthaft beeinträchtigen und die Beschränkung für die Erfüllung der Forschungs- oder Statistikzwecke notwendig ist. Das Recht auf Auskunft gemäß Artikel 15 der Verordnung (EU) 2016/679 besteht darüber hinaus nicht, wenn die Daten für Zwecke der wissenschaftlichen Forschung erforderlich sind und die Auskunftserteilung einen unverhältnismäßigen Aufwand erfordern würde.

(3) Ergänzend zu den in $\$ 22$ Absatz 2 genannten Maßnahmen sind zu wissenschaftlichen oder historischen Forschungszwecken oder zu statistischen Zwecken verarbeitete besondere Kategorien personenbezogener Daten im Sinne des Artikels g Absatz 1 der Verordnung (EU) 2016/679 zu anonymisieren, sobald dies nach dem Forschungs- oder Statistikzweck möglich ist, es sei denn, berechtigte Interessen der betroffenen Person stehen dem entgegen. Bis dahin sind die Merkmale gesondert zu speichern, mit denen Einzelangaben über persönliche oder sachliche Verhältnisse einer bestimmten oder bestimmbaren Person zugeordnet werden können. Sie dürfen mit den Einzelangaben nur zusammengeführt werden, soweit der Forschungsoder Statistikzweck dies erfordert.

(4) Der Verantwortliche darfpersonenbezogene Daten nur veröffentlichen, wenn die betroffene Person eingewilligt hat oder dies für die Darstellung von Forschungsergebnissen über Ereignisse der Zeitgeschichte unerlässlich ist.“

Bei der Überarbeitung der Forschungsklauseln in den Landesdatenschutzgesetzen verfolgten die Länder keine gemeinsame Linie. Die Anpassungen an die DSGVO beschränken sich bisher weitgehend auf die Terminologie. Wurde auf die in der DSGVO vorgesehenen Privilegierungen Bezug genommen, so zumeist zur Einschränkung der Betroffenenrechte. Die Regelungen der Bundesländer zur Sekundärnutzung von Daten für Forschungszwecke schreiben weitgehend das bisher geltende Recht fort: $\mathbb{s} 13$ LDSG BW, Art. 25 BayDSG, $\mathbb{s} 17$, 35 BlnDSG, $\mathbb{2} 25$ BbgDSG, $\mathbb{1} 13$ BremDSGVOAG,

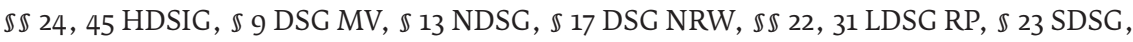
$\mathbb{S} 12$ SächsDSG, $\mathbb{S} 27$ DSG LSA, $\mathbb{S} 13,26$ LDSG SH, $\mathbb{2} 28$ ThürDSG. ${ }^{175}$

Für Sozialleistungsträger ( $\$ 12$ SGB I i.V.m. $\mathbb{S} 18-29$ SCB I) gilt bzgl. der Datenverarbeitung für Forschungszwecke nicht $\$ 27$ BDSG, sondern gelten allgemein die Regelungen des $\mathbb{7} 75$ SGB X, soweit es um die Übermittlung an Dritte geht, sowie die \$s 67b, 67c SCB X in Bezug auf Eigenforschung. ${ }^{176}$

175 Bernhardt/Ruhmann/Weichert mit einer Dokumentation sowie einer vergleichenden Bewertung; BKL-R, 211ff.; Graf von Kielmansegg in TMF, $103 f$.

176 Angepasst an die DSGVO mit G. v. 17.07.2017, BGBI. I S. 2541; ausführlich dazu Schäfer in Kipker/Voskamp, 314ff. 
\$ 75 SCB X zur Übermittlung für Forschungszwecke hat folgenden Wortlaut:

„(1) Eine Übermittlung von Sozialdaten ist zulässig, soweit sieerforderlich ist für ein bestimmtes Vorhaben

1. der wissenschaftlichen Forschung im Sozialleistungsbereich oder der wissenschaftlichen Arbeitsmarkt- und Berufsforschung oder

2. der Planung im Sozialleistungsbereich durch eine öffentliche Stelle im Rahmen ihrer Aufgaben

und schutzwürdige Interessen der betroffenen Person nicht beeinträchtigt werden oder das öffentliche Interesse an der Forschung oder Planung das Geheimhaltungsinteresse der betroffenen Person erheblich überwiegt. Eine Übermittlung ohne Einwilligung der betroffenen Person ist nicht zulässig, soweit es zumutbar ist, ihre Einwilligung einzuholen. Angaben über den Namen und Vornamen, die Anschrift, die Telefonnummer sowie die für die Einleitung eines Vorhabens nach Satz 1 zwingend erforderlichen Strukturmerkmale der betroffenen Person können für Befragungen auch ohne Einwilligungen übermittelt werden. Der nach Absatz 4 Satz 1 zuständigen Behörde ist ein Datenschutzkonzept vorzulegen.

(2) Ergibt sich aus dem Vorhaben nach Absatz 1 Satz 1 eine Forschungsfrage, die in einem inhaltlichen Zusammenhang mit diesem steht, können hierzu auf Antrag die Frist nach Absatz 4 Satz 5 Nummer 4 zur Verarbeitung der erforderlichen Sozialdaten verlängert oder eine neue Frist festgelegt und weitere erforderliche Sozialdaten übermittelt werden.

(3) Soweit nach Absatz 1 oder 2 besondere Kategorien von Daten im Sinne von Artikel g Absatz 1 der Verordnung (EU) 2016/679 an einen Dritten übermittelt oder nach Absatz 4a von einem Dritten verarbeitet werden, sieht dieser bei der Verarbeitung angemessene und spezifische Maßnahmen zur Wahrung der Interessen der betroffenen Person gemäß 22 Absatz 2 Satz 2 des Bundesdatenschutzgesetzes vor. Ergänzend zu den dort genannten Maßnahmen sind die besonderen Kategorien von Daten im Sinne des Artikels g Absatz 1 der Verordnung (EU) 2016/679 zu anonymisieren, sobald dies nach dem Forschungszweck möglich ist.

(4) Die Übermittlung nach Absatz 1 und die weitere Verarbeitung sowie die Übermittlung nach Absatz 2 bedürfen der vorherigen Genehmigung durch die oberste Bundes- oder Landesbehörde, die für den Bereich, aus dem die Daten herrühren, zuständig ist. Die oberste Bundesbehörde kann das Genehmigungsverfahren bei Anträgen von Versicherungsträgern nach 11 Absatz 1 Satz 1 des Vierten Buches oder von deren Verbänden aufdas Bundesversicherungsamt übertragen. Eine Übermittlung von Sozialdaten an eine nicht-öffentliche Stelle und eine weitere Verarbeitung durch diese nach Absatz 2 darfnurgenehmigt werden, wenn sich die nichtöffentliche Stelle gegenüber der Genehmigungsbehörde verpflichtet hat, die Daten nur für den vorgesehenen Zweck zu verarbeiten. Die Genehmigung darfim Hinblick aufdie Wahrung des Sozialgeheimnisses nur versagt werden, wenn die Voraussetzungen des Absatzes 1, 2 oder 4a nicht vorliegen. Sie muss

1. den Dritten, an den die Daten übermittelt werden,

2. die Art der zu übermittelnden Sozialdaten und den Kreis der betroffenen Personen,

3. die wissenschaftliche Forschung oder die Planung, zu der die übermittelten Sozialdaten verarbeitet werden dürfen, und

4. den Tag, bis zu dem die übermittelten Sozialdaten verarbeitet werden dürfen, 
genau bezeichnen und steht auch ohne besonderen Hinweis unter dem Vorbehalt der nachträglichen Aufnahme, Änderung oder Ergänzung einer Auflage. Nach Ablauf der Frist nach Satz 5 Nummer 4 können die verarbeiteten Daten bis zu zehn Jahre lang gespeichert werden, um eine Nachprüfung der Forschungsergebnisse aufder Grundlage der ursprünglichen Datenbasis sowie eine Verarbeitung für weitere Forschungsvorhaben nach Absatz z zu ermöglichen.

(4a) Ergänzend zur Übermittlungvon Sozialdaten zu einem bestimmten Forschungsvorhaben nach Absatz 1 Satz 1 kann die Verarbeitung dieser Sozialdaten auch für noch nicht bestimmte, aber inhaltlich zusammenhängende Forschungsvorhaben des gleichen Forschungsbereiches beantragt werden. Die Genehmigung ist unter den Voraussetzungen des Absatzes 4 zu erteilen, wenn sich der Datenempfänger gegenüber der genehmigenden Stelle verpflichtet, auch bei künftigen Forschungsvorhaben im Forschungsbereich die Genehmigungsvoraussetzungen einzuhalten. Die nach Absatz 4 Satz 1 zuständige Behörde kann vom Antragsteller die Vorlage einer unabhängigen Begutachtung des Datenschutzkonzeptes verlangen. Der Antragsteller ist verpflichtet, der nach Absatz 4 Satz 1 zuständigen Behörde jedes innerhalb des genehmigten Forschungsbereiches vorgesehene Forschungsvorhaben vor dessen Beginn anzuzeigen und dabei die Erfüllung der Genehmigungsvoraussetzungen darzulegen. Mit dem Forschungsvorhaben darfacht Wochen nach Eingang der Anzeige bei der Cenehmigungsbehörde begonnen werden, sofern nicht die Genehmigungsbehörde vor Ablauf der Frist mitteilt, dass für das angezeigte Vorhaben ein gesondertes Genehmigungsverfahren erforderlich ist.

(5) Wird die Verarbeitung von Sozialdaten nicht-öffentlichen Stellen genehmigt, hat die genehmigende Stelle durch Auflagen sicherzustellen, dass die der Cenehmigung durch Absatz 1, 2 und 4 a gesetzten Grenzen beachtet werden.

(6) Ist der Dritte, an den Sozialdaten übermittelt werden, eine nicht-öffentliche Stelle, unterliegt dieser der Aufsicht der gemäß 40 Absatz 1 des Bundesdatenschutzgesetzes zuständigen Behörde.“

Die Zweckänderung für eigene Forschungszwecke für Sozialleistungsträger ist in \$ 67c Abs. 2 Nr. 2 SGB X geregelt (s.u. Kap. 8.4). Die einwilligungsbasierte Datenverarbeitung findet in $\$ 67 \mathrm{~b}$ SGB X eine allgemeine Regelung (s.u. Kap. 7.4). Die Weiterverarbeitung von Sozialdaten, die für Forschungszwecke gespeichert sind, ist in $\$ 67 \mathrm{C}$ Abs. 5 SGB X geregelt (s.u. Kap. 8.4).

Neben den allgemeinen Forschungsregelungen im SCB X bestehen in den speziellen Sozialgesetzbüchern spezifische Regelungen. Die gilt für die gesetzliche Krankenversicherung mit einigen Grundregeln in $\$ \mathbb{2} 287$, 303a-303f, 363 SGB V sowie einer Vielzahl von weiteren Vorgaben im SGB V (z.B. $\$ \$ 25$ Abs. 5, 63 Abs. 1 S. 1 Nr. 8, 68a Abs. 3 Nr. 3, 137a, 117 Abs. 1 u. 2, 287a, 327 Abs. 1 Nr. 1). Weitere Forschungsregelungen finden sich z.B. zu Daten aus der gesetzlichen Unfallversicherung ( $\$ 9$ Abs. 8 SCB $\mathrm{VII})^{177}$, zur Arbeitsmarkt- und Berufsforschung sowie zur Wirkungsforschung in der Grundsicherung ( $\$ \$ 280-282$ SGB III, $\mathbb{5} 55$ SGB II $)^{178}$ oder zu Daten aus der Pflegeversicherung ( $\$ 98 \mathrm{SGB} X \mathrm{XI})$.

Soweit bereichsspezifische Forschungsregelungen im Bundes- und Landesrecht bestehen, gehen diese gemäß $\mathbb{1}$ Abs. 2 BDSG $^{179} \mathrm{bzw}$. generell gemäß dem Vorrang der

177 Schäfer in Kipker/Voskamp, $385 \mathrm{ff}$.

178 Schäfer in Kipker/Voskamp, $402 \mathrm{ff}$.

179 Für das Sozialrecht $\$ 35$ Abs. 2a SGB I. 
spezielleren Regelung den allgemeinen Forschungsklauseln vor. Solche Normen finden sich in vielen Bereichen des Sozialrechts ( $\$ 287$ SGB V, $\mathbb{s} 98$ SGB XI, $\mathbb{1} 119$ SGB XII), im Strafprozessrecht ( $\$ 476$ StPO), in $\$ 42 a$ BZRG, im Strafvollzugsrecht ( $\$ 186$ StVollzG). Für die Ärzteschaft gelten die Berufsordnungen der Landesärztekammern, die sich weitgehend an $\mathbb{1 5}$ MBOÄ orientieren bzw. damit identisch sind. ${ }^{180}$ Im Medizinbe-

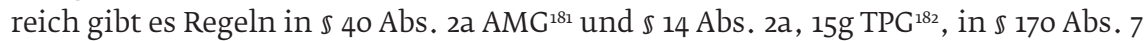
StrlSchG, in den Psychisch-Kranken- ${ }^{183}$ den Krankenhaus- ${ }^{-184}$ und den Krebsregistergesetzen ${ }^{185}$, nicht aber z.B. im Gendiagnostikrecht ( $\$ 2$ Abs. 2 Nr. 1 GenDG). ${ }^{186}$

Nicht eindeutig ist, wie sich die Forschungsregelungen in Bund und Ländern auf die Öffnungsklauseln der DSGVO beziehen. Teilweise beschränkt sich die Anwendung der dortigen materiell-rechtlichen Regelungen auf sensitive Daten nach Art. 9 Abs. 1 DSGVO, so etwa in $\$ 27$ Abs. 1 BDSG. ${ }^{187}$ Dem liegt die Erwägung zugrunde, dass die Art. 5, 6 DSGVO in Bezug auf nicht-sensitive Daten abschließend sind. ${ }^{188}$ Die meisten Landesgesetzgeber regelten dagegen generell die Verarbeitung von Daten für Forschungszwecke „einschließlich solcher nach Artikel g Absatz 1 der Verordnung (EU) 2016/619“. ${ }^{889}$ Für die Regulierung der Verarbeitung von nicht-sensitiven Daten können die Öffnungsklauseln in Art. 6 Abs. 2, 85 Abs. 1 und 89 DSGVO geltend gemacht werden (s.o. Kap. 4.1).

Es bleibt aber fraglich, inwieweit die darin enthaltenen Regelungen mit den materiell-rechtlichen Vorgaben in den Art. 5 Abs. 1 lit. b, 6 Abs. 4 DSGVO zur Zweckprivilegierung bzw. Zweckvereinbarkeit in Einklang zu bringen sind. ${ }^{190}$ Einige Gesetze sehen eine Festlegung auf ein „bestimmtes Forschungsvorhaben“ vor. ${ }^{191}$ Verbundprojekte sind mit dieser Formulierung nur schwerlich in Einklang zu bringen, ebenso Projekte, in denen die wissenschaftlichen Fragestellungen zu Forschungsbeginn noch nicht endgültig feststehen und die zeitlich nicht begrenzt sind (z.B. Register). In einigen Gesetzen wird die Zweckänderung im Rahmen einer Sekundärnutzung davon abhängig gemacht, dass die Verarbeitungszwecke gegenüber den Betroffeneninteressen „überwiegen“", ${ }^{192}$ zumeist ist aber nötig, dass diese „erheblich überwiegen“. ${ }^{193}$

180 Schneider, Sekundärnutzung klinischer Daten - Rechtliche Rahmenbedingungen, Schriftenreihe der Technologie- und Methodenplattform für die vernetzte medizinische Forschung e.V. (TMF) 2015, $295 \mathrm{ff.}$

181 Schneider 2015, 64ff.

182 Schneider 2015, 61.

183 Schneider 2015, 72.

184 Übersicht bei Dierks 2019, 37ff.; vgl. Schneider 2015, $244 f$.

185 Gode/Niemeck in Kipker/Voskamp, 539f.; Sachverständigenrat, 206.

186 Schneider 2015, 55.

187 Ebenso \$ 24 Abs. 1 S. 1 HDSIG, $\$ 22$ Abs. 1 LDSG RP.

188 Bernhardt/Ruhmann/Weichert, 5.

$189 § 17$ Abs. 1 BlnDSG, $\ 25$ Abs. 1 S. 1 BbgDSG, $\ 11$ Abs. 1 HmbDSG, $\ 9$ DSG Abs. 1 S. 1 M-V, $\ 13$ Abs. 1 S. 1 NDSG, $\S 17$ Abs. 1 DSG NRW, $§ 22$ Abs. 1 S. 1 DSG Saar, $\ 12$ Abs. 1 SächsDSDG, $\ 13$ Abs. 1, S. 1 LDSG SH, $\ 28$ ThürDSG, wohl auch Art. 25 BayDSG.

190 Bernhardt/Ruhmann/Weichert, $5 f$.

191 \25 Abs. 1 BbgDSG, \ 11 Abs. 1 HmbDSG, \$ 9 Abs. 1 S. 1 DSG M-V, \$ 13 Abs. 1 NDSG, \ 23 Abs. 1 S. 1 DSG Saar, unklar $\$ 22$ Abs. 1 LDSG RP.

192 \13 Abs. 1 S. 1 LDSG BW, $§ 25$ Abs. 1 S. 1 BbgDSG, $§ 24$ Abs. 1 S. 1 HDSIG, $\ 13$ Abs. 1 S. 1 NDSG, $\ 17$ Abs. 1 DSG NRW, $\ 12$ Abs. 1 SächsDSDG.

193 \ 27 Abs. 1 S. 1 BDSG, \9 Abs. 1 S. 1 DSG M-V, \22 Abs. 1 LDSG RP, \$ 23 Abs. 1 S. 1 DSG Saar, keine Regelung BayDSG, ThürDSG; zur Auslegung BKL-R, $207 f$. 
Unstreitig ist, dass sich das Recht der Mitgliedstaaten in dem von der DSGVO vorgezeichneten Rahmen halten muss. Solange keine höchstrichterliche Klärung über die Rechtsgrundlagen für Forschungszwecke vorliegt, ist es geboten, jeweils die spezifischste Norm heranzuziehen, auch wenn es sich hierbei um eine Regelung des deutschen Bundes- oder des Landesrechts handelt. Diese muss dann aber in jedem Fall im Lichte der Vorgaben der DSGVO ausgelegt werden und im Zweifel bei einem Verstoß hiergegen unangewendet bleiben (s.u. Kap. 8.4). ${ }^{194}$

Liegt eine wirksame Einwilligung vor, so kann hierauf Bezug genommen werden (s.u.). Fehlt es hieran, so ist auf eine gesetzliche Grundlage zurückzugreifen. Dabei muss in jedem Fall eine Abwägung vorgenommen werden zwischen den Schutzinteressen der Betroffenen und den Interessen an dem konkreten Forschungsvorhaben mit den konkreten Daten. ${ }^{195}$

194 Heberlein in Ehmann/Selmayr, Art. 6 Rn. 64, 71; Hornung/Spiecker in SHS, Einl Rn. 265; Ruffert in Callies/Ruffert, Art. 1 AEUV Rn. 24.

195 Zu den unterschiedlichen Abwägungsklauseln, der Offenheit der Abwägung und Abwägungsaspekten Graf von Kielmansegg in TMF, $106 \mathrm{ff}$. 